\title{
Review Article Newly Identified Mechanisms of Total Parenteral Nutrition Related Liver Injury
}

\author{
Ajay Kumar Jain ${ }^{1,2}$ and Jeffrey H. Teckman ${ }^{1}$ \\ ${ }^{1}$ St. Louis University School of Medicine, Cardinal Glennon Children's Medical Center, 1465 South Grand Boulevard, \\ St. Louis, MO 63104, USA \\ ${ }^{2}$ Department of Pediatrics, Saint Louis University, SSM Cardinal Glennon Hospital, 1465 South Grand Boulevard, \\ St. Louis, MO 63104, USA \\ Correspondence should be addressed to Ajay Kumar Jain; ajain3@slu.edu
}

Received 4 March 2014; Accepted 18 May 2014; Published 25 June 2014

Academic Editor: Sharon DeMorrow

Copyright (C) 2014 A. Kumar Jain and J. H. Teckman. This is an open access article distributed under the Creative Commons Attribution License, which permits unrestricted use, distribution, and reproduction in any medium, provided the original work is properly cited.

\begin{abstract}
Total parenteral nutrition (TPN), a lifesaving therapy, involves providing nutrition by bypassing the gut. Unfortunately it is associated with significant complications including gut atrophy and parenteral nutrition associated liver disease (PNALD). PNALD includes steatosis, cholestasis, disrupted glucose metabolism, disrupted lipid metabolism, cirrhosis, and liver failure. The etiopathogenesis remains poorly defined; however, an altered enterohepatic circulation, disrupting nuclear receptor signaling, is emerging as a promising mechanism. Rodent models and our piglet TPN model have shown that, during regular feeding, bile acids activate farnesoid X receptor (FXR) in the gut and enhance fibroblast growth factor 19 (FGF19) level. FGF19 regulates bile acid, lipid, and glucose metabolism. We noted reduced FGF19 with TPN use and substantial improvement in FGF19, bilirubin, and metabolic profiles with the FXR agonist chenodeoxycholic acid (CDCA). Additionally, CDCA caused gut growth and enhanced expression of glucagon like peptides (GLPs). GLPs regulate gut trophic effects, insulin, glucose homeostasis, and hepatic steatosis. GLP secretion is regulated by the CDCA activated receptor TGR5. This leads to an important conclusion that, in addition to a disrupted FXR-FGF19 axis, a disrupted TGR5-GLP axis may contribute to TPN related pathologies. Thus modulators of FXR-FGF19 and the TGR5-GLP axis could help bring forward novel treatment strategies.
\end{abstract}

\section{Introduction}

Parenteral nutrition has been in use since the 1960s [1, 2]. Total parenteral nutrition (TPN) involves providing all of patient's nutritional needs intravenously and is lifesaving in clinical settings in which adequate enteral based delivery of such nutrition is not possible. It has been one of the most promising modalities of nutrition in neonates, older pediatric patients, and adults with lost or impaired gut function. TPN infusion therapy has grown enormously over the last few decades. There are tens of thousands of patients worldwide permanently dependent on parenteral nutrition (PN) for survival [3]. In addition, there are several fold higher numbers of patients requiring $\mathrm{PN}$ for varying duration during hospital stay or home care. Unfortunately, it is also associated with important complications and significant morbidity and mortality, including the well-recognized parenteral nutrition associated liver disease (PNALD) which includes steatosis, cholestasis, disrupted glucose metabolism, disrupted lipid metabolism, cirrhosis, development of portal hypertension, and ultimately liver failure $[4,5]$. The mechanisms of such injury remain poorly defined.

Despite multiple theories being advanced, the etiology and pathophysiology of PNALD remain elusive. Among the various possible mechanisms, a lack of enteral feeding resulting in gut atrophy and the disruption of enterohepatic circulation has been proposed as a contributor to PNALD $[6,7]$. 
Using a piglet animal TPN model, we have documented TPN induced liver and gut pathologies. We have also described the role of an altered enterohepatic circulation and disrupted signaling of bile acids contributing to such injury. This shall be the focus of this review.

1.1. Lack of Enteral Feeding. It is commonly believed that once cholestasis sets in, its reversal occurs only when a patient is receiving all or a major portion of the calories enterally. In fact, enteral nutrition helps to protect against the development of PNALD and cholestasis as noted in several studies [8-10]. In TPN model systems we have shown significantly elevated bilirubin and PNALD in animals on TPN versus those getting enteral feeds [8]. As reported in the literature, we have also noted significant hepatocellular dysfunction, elevated hepatic triglycerides, and steatosis with TPN use [11-13].

Numerous studies including results from our model have shown that TPN use also induces gut mucosal atrophy, which has been attributed possibly to the absence of trophic signals released in response to luminal nutrients, during regular feeding $[14,15]$. However, the physiologic nature and relative importance of these trophic signals have yet to be established [16]. We noted significant gut atrophy within 2 weeks of TPN infusion [8]. However, recent evidence suggests that gut atrophy might be occurring much sooner. In a study using an animal TPN model, mucosal atrophy was found to occur as early as 24 hours in the jejunum as evident by a reduction in villous height and protein synthesis [17]. Given this evidence, it is believed that the underlying adaptation to TPN might occur much earlier, due to the high metabolic rate and rapid turnover of intestinal epithelial cells.

Thus it is possible that a lack of enteral feeding, which is known to disrupt normal enterohepatic circulation, may be a contributor to TPN associated pathologies as documented in our previously published data [8].

Normal enterohepatic circulation depends on normal synthesis, conjugation, secretion, and recirculation of bile acids. This brings to attention an important question whether there is a homeostatic equilibrium of certain signaling molecules between the gut and the liver, a disruption of which leads to PNALD. This has taken special importance after the establishment of the existence of the nuclear receptor farnesoid X receptor (FXR) [18]. Several studies have shown that bile acids are physiological ligands for FXR. FXR is abundant in liver and gut tissue [19]. It is also well established that activation of FXR is noted with the primary bile acid chenodeoxycholic acid (CDCA), a potent FXR agonist. Bile acids have also been known to have a trophic effect on gut growth and integrity $[20,21]$. We have shown significant gut growth and amelioration of atrophy with enteral bile acid treatment [8].

\subsection{Bile Acid Mediated FXR Induction of Fibroblast Growth Factor 19 (FGF19)}

1.2.1. FGF19 Production. During normal enterohepatic circulation, bile acid absorption in the ileum is associated with activation of FXR which has been shown to exert several important regulatory effects on lipid and carbohydrate metabolism.

In fact, recent insights from cell culture and animal models show that enteral bile acids robustly activate FXR in intestinal epithelial cells $[8,22,23]$. Such activation stimulates production of a growth factor, fibroblast growth factor 15 (FGF15) and its human orthologue fibroblast growth factor 19 (FGF19) which is delivered via the portal circulation to the liver [24]. We have assayed FGF19 in TPN versus enteral fed animals and found robust upregulation of FGF19 upon enteral bile acid treatment, with significant reduction upon TPN infusion [8].

1.2.2. Hepatic Bile Acids and Cholesterol 7 Alpha Hydroxylase (CYP7A1). In the liver, CYP7A1, which is a rate limiting step for hepatic bile acid synthesis, is suppressed via the short heterodimer partner- (SHP-) liver receptor homologue 1 (LRH1) cascade $[25,26]$ and by mechanisms independent of SHP gene induction [27, 28]. Rodent studies and recently our work in a TPN piglet model have indicated that intestinal FGF19 may function as a secretory signal to the liver that contributes to intestinal FXR dependent repression of CYP7A1 [29]. In order to determine whether fasting serum levels of FGF19 were changed with bile acid regulated enterohepatic circulation, Lundåsen et al. assayed FGF19 levels in serum samples from subjects treated with the bile acid resin cholestyramine (which sequesters BAs preventing their absorption) and subjects treated with CDCA. Bile acid synthesis was determined by the serum levels of 7-alphahydroxy-4-cholesten-3-one (C4), a marker for CYP7A1 enzymatic activity. Treatment with cholestyramine led to an 18fold increase in serum $\mathrm{C} 4$ and a reduction in FGF19 levels by $87 \%$. In the CDCA treatment group, FGF19 levels increased by $250 \%$ while serum C4 levels decreased by $26 \%$ [30].

Importantly, FGF19 is expressed in the small intestine [31] but not in the liver $[32,33]$. In a study by Kim et al., tissuespecific roles of FXR were examined. Tissue-specific FXR null mouse models for liver (FxrL) and intestine (FxrIE) were used. Bile acid pool size was increased with FXR deficiency in either liver FxrL or intestine FxrIE. Treatment of these mice with GW4064 a FXR-selective agonist significantly repressed CYP7A1 in FxrL mice but not in FxrIE mice. This demonstrated that the repression of CYP7A1 was mediated primarily by FXR activation in the intestine and not in the liver [34].

1.2.3. Glucose and Lipid Metabolism. Fu et al. have shown that, in obese mice resulting from leptin deficiency or genetic ablation of brown adipose tissue, treatment with human fibroblast growth factor 19 prevented or reversed diabetes and improved glycemic control. They also showed that there was a reduction in triglyceride levels in the FGF19 treated mice. Liver expression of leptin receptor and expression of acetyl coenzyme A carboxylase were acutely decreased with FGF19 [35]. We have also previously shown that TPN infusion, in addition to decreasing FGF19 levels, results in insulin resistance. Additionally hypertriglyceridemia was noted with 
TPN infusion [8]. FGF receptor 4 (FGFR4) which is abundant in the liver is a receptor for FGF19 and FGF15 [36]. Mice lacking FGFR4 have an increased bile acid pool [36, 37]. An increased white adipose tissue mass, glucose intolerance, insulin resistance, hyperlipidemia, and hypercholesterolemia were seen by Huang et al. in FGFR4 deficient mice that were fed a normal diet, indicating an important role of FGF19 in glucose and lipid metabolic regulation [38].

Ursodeoxycholic acid (UDCA) has been used in patients with TPN cholestasis with inconsistent results. Heubi et al. in their study in 22 infants on TPN, who received oral tauroursodeoxycholic acid (TUDCA) against controls, documented no difference in peak serum conjugated bilirubin, alanine aminotransferase, alkaline phosphatase, or bile acid levels [39]. Reihner et al. in their study on 61 human subjects noted significant suppression of CYP7A1 on treatment with CDCA at $15 \mathrm{mg} / \mathrm{kg} /$ day. No change in CYP7A1 levels was noted in the UDCA treated patients [40]. It is particularly important to know that the commonly used bile acids UDCA and TUCDA have minimal activity for FXR. Park et al. showed that while CDCA was a strong agonist for FXR, no activation of FXR was seen with UDCA [41]. Similar results were reported by Makishima et al. in 1999 [20]. We have also shown significant improvement in PNALD with CDCA infusion [8]. This pathway for the FXR-FGF19 axis during TPN infusion is schematically depicted in Figure 1.

The above seem to be some of the direct effects of an altered enterohepatic circulation leading to altered nuclear receptor signaling. We believe that the disrupted enterohepatic circulation also leads to several other nuclear receptor dependent pathways to become abnormal which contribute to TPN pathologies.

1.3. Bacterial Overgrowth, Endotoxin, and Inflammation. Intestinal hypomotility as a consequence of starvation promotes bacterial overgrowth in the small intestine [42]. On morphometric analysis we have shown severe villous blunting and significant reduction in the villous/crypt ratios with TPN infusion. This was most prominent in the proximal small bowel. In fact grossly the small bowel was thin and friable in TPN versus enteral feeding [8]. Such atrophy has been reported in other studies and it is possible that the atrophic gut contributes to increased permeability $[16,17]$.

Bacterial permeation across the gut wall increases with TPN use, likely worsened by bacterial overgrowth. This results in endotoxin associated downregulation of bile acid transport [43]. Neonates with short-bowel syndrome are particularly susceptible to both bacterial translocation and parenteral nutrition induced hepatic injury and thus occurrence of small-bowel bacterial overgrowth is concerning [44]. Lichtman et al. created small-bowel bacterial overgrowth in a rat model using jejunal self-filling blind loops. The animals developed weight loss, hepatomegaly, and hepatic inflammation as early as 4 weeks after the creation of the selffilling bowel loops. Subsequently they reported evidence of biliary tract injury in these rats with decreased bile flow rates, dilatation of bile ducts, and beaded appearance of the bile ducts on cholangiogram [45].
Multiple investigators have reported a decrease in inflammation induced liver injury in rats after antibiotic initiation of oral tetracycline and/or metronidazole, which indirectly suggests a role of bacteria in the development or exacerbation of PNALD [46]. Whiting et al. illustrated the role of endotoxin and tumor necrosis factor (TNF) in sepsis mediated cholestasis [47]. Other inflammatory or sepsis-related conditions like inflammatory bowel disease have been shown to contribute to the development of cholestasis $[48,49]$. Both clinical and animal studies have demonstrated the role of cytokines in the development of PNALD. In rat studies, Zheng et al. found significantly higher levels of TNF and interleukin6 (IL-6) in rats that received parenteral nutrition (PN) than in a control group receiving normal chow plus saline infusion. The PN group also had significantly higher levels of endotoxin in their portal blood [50]. Elevated cytokine levels were postulated to occur in response to bacterial translocation. Though treatment directed against cytokines helps in decreasing cholestasis, given the above evidence we believe that further studies are needed to definitively establish the role of these mediators in the development of PNALD. In one study, treatment with anti-TNF antibody resulted in improvement in a patient with PNALD [51].

1.4. Role of Nuclear Receptors in Bacterial Growth and Responses. Tumor necrosis factor and interleukin $1 \beta$ (IL- $1 \beta)$, which are proinflammatory cytokines, have been shown to downregulate transcription of bile acid transporter, sodiumtaurocholate cotransporting polypeptide (NTCP), via inhibition of the nuclear retinoic acid and retinoid $\mathrm{X}$ receptor heterodimer (RXR:RAR) in rats [52]. A downregulation of hepatic bile acid transporters, bile salt export pump (BSEP) and multidrug resistance protein 2 (MRP2), was noted by Hojo et al. after intravenous administration of lipopolysaccharide [53]. Studies in mice using intraperitoneal lipopolysaccharide administration showed reduced expression of the mRNAs of bile acid transporters, including NTCP and BSEP [54]. FXR has been shown to induce genes involved in gut protection and inhibit bacterial overgrowth and mucosal injury in ileum by Inagaki et al. Mice lacking FXR were shown to have increased ileal levels of bacteria and a compromised epithelial barrier [55]. In addition, FXR regulates endothelial nitric oxide synthase (eNOS), carbonic anhydrase 12 (CAR12), and angiogenin (ANG1) involved in gut inflammation, bacterial growth inhibition, and mucosal injury. Mice lacking FXR have compromised gut permeability on fluorescein isothiocyanate- (FITC-) conjugated dextran assay [56]. We have noted significant improvement in gut morphology with bile acid infusion in TPN infused animals [8]. Since bile acids are physiological ligands for FXR, there appears to be a central role for FXR in protecting the small intestine from bacterial invasion. It was also suggested that FXR agonists may prevent epithelial deterioration and bacterial translocation in patients with impaired bile flow. Our recent studies have shown a striking and robust gut growth with CDCA in a TPN piglet model [8]. Glucagon-like peptides (GLPs) which are thought to regulate gut trophic effects (GLP-2), insulin, and glucose metabolism (GLP-1) 


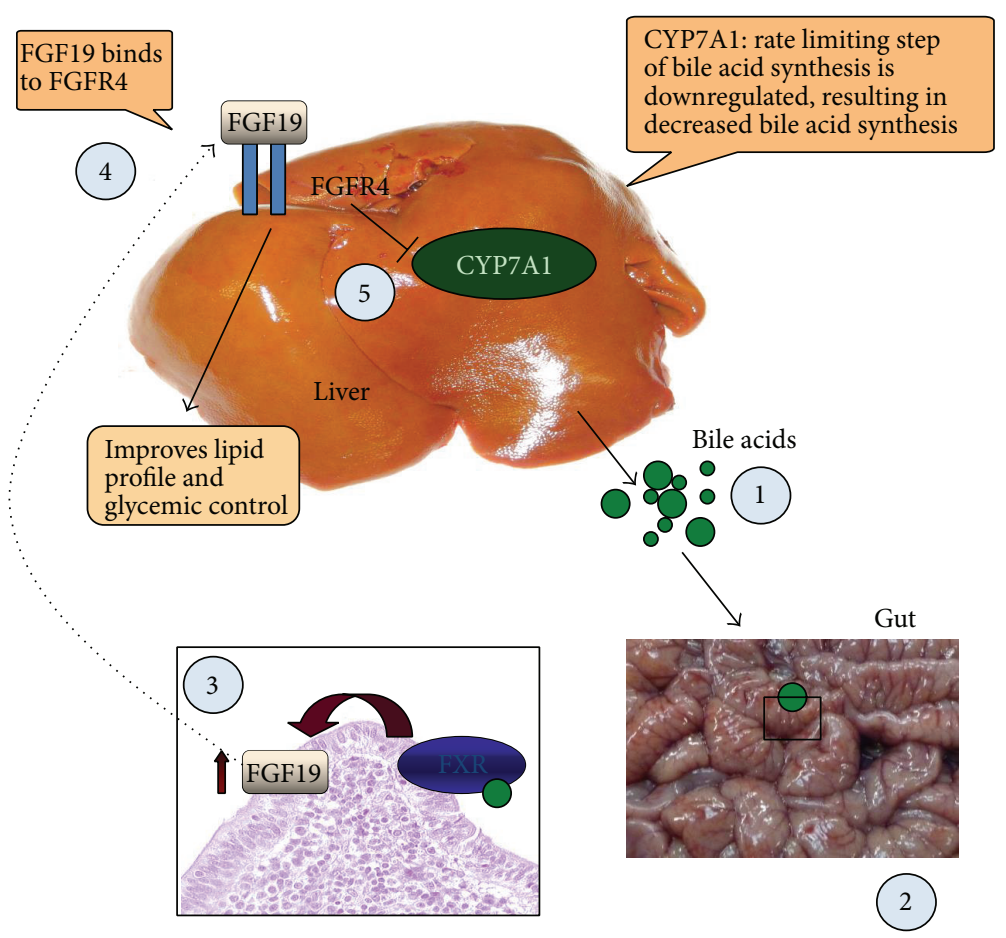

FIGURE 1: Schematic representation. (1) Bile acids (BA) secreted into bile. (2) BA reach terminal ileum. (3) BA cause secretion of FGF19 upon FXR stimulation. (4) FGF19 travels through the portal circulation to liver, binding to its receptor FGFR4. (5) Hepatic effects include suppression of CYP7A1, improvement in lipid profile, and glycemic control. FGFR4: fibroblast growth factor receptor 4; CYP7A1: cholesterol 7 alpha hydroxylase; FXR: farnesoid X receptor; FGF19: fibroblast growth factor 19.

were upregulated with CDCA in our TPN animal model [8]. GLP-1 has also been shown to modulate hepatic steatosis. As the GLPs are modulated via the CDCA activated G-protein coupled receptor TGR5, we postulate that at least partial improvement in PNALD could result from a direct effect of CDCA on TGR5 activation [16].

1.5. Role of Lipids in PNALD. There has been recent interest in fish oil based fat emulsions to improve TPN related pathologies. Though outside the purview of this review, we shall briefly discuss such an approach. Intralipid (FreseniusKabi) is the standard lipid emulsion for intravenous use in the US for TPN nutrition. Intralipid, which is produced from soybean oil, contains a high ratio $(7: 1)$ of $n-6$ to $n-$ 3 polyunsaturated fatty acids (PUFA). Phytosterols present in soy derived lipid emulsions are known FXR antagonists [57]. Recently Omegaven which has a very low ratio $(1: 8)$ of n-6 to n-3 PUFAs and is derived from fish oil has shown promise in several clinical studies. It has been shown that n-3 fatty acid solutions decrease de novo lipogenesis and prevent or attenuate $\mathrm{PN}$-associated steatosis and cholestasis $[58,59]$. Two $n-3$ fatty acids present in Omegaven are docosahexaenoic acid (DHA) and eicosapentaenoic acid (EPA). Peroxisome proliferator-activated receptor (PPAR) and liver $\mathrm{X}$ receptor (LXR) are nuclear receptors involved in bile and fat metabolism, which are known to be modulated by DHA [60]. Additionally, lipogenesis is inhibited by DHA and EPA via inhibition of the lipogenesis transcription factor, sterol regulatory element binding protein- (SREBP-) 1c [61-63]. Bile salt transporters like BSEP are modulated via FXR and LXR which in turn are under regulatory control by n-3 PUFA. It is thus predicted that n-3 PUFA rich fat emulsions may alter the course of PNALD [64-66].

\section{Conclusion}

We conclude from rodent studies and our recent results from a TPN model that an altered enterohepatic circulation during TPN infusion contributes to TPN pathologies. This is the result of an alteration of the FXR-FGF19 axis and likely the TGR5-GLP axis. CDCA which is a physiological ligand for FXR exerts beneficial effects as noted in our TPN animal model. Such improvement is through activation of FGF19 upon FXR stimulation. Additionally, there is increased GLP secretion with activation of the CDCA activated G proteincoupled receptor TGR5. New literature is presenting very promising data on the role of bile acids and their regulation of hepatic steatosis, lipid, and glucose metabolism. In fact both FXR agonist and TGR5 agonists are being used in clinical studies for hepatic disorders. It is quite intuitive that further research evaluating such agonists in clinical human studies may bring a paradigm change in our approach to treating TPN related pathologies. 


\section{Conflict of Interests}

The authors declare that there is no conflict of interests regarding the publication of this paper.

\section{References}

[1] S. J. Dudrick, D. W. Wilmore, H. M. Vars, and J. E. Rhoads, "Long-term total parenteral nutrition with growth, development, and positive nitrogen balance," Surgery, vol. 64, no. 1, pp. 134-142, 1968.

[2] S. J. Dudrick, "Early developments and clinical applications of total parenteral nutrition," Journal of Parenteral and Enteral Nutrition, vol. 27, no. 4, pp. 291-299, 2003.

[3] L. Howard, M. Ament, C. R. Fleming, M. Shike, and E. Steiger, "Current use and clinical outcome of home parenteral and enteral nutrition therapies in the United States," Gastroenterology, vol. 109, no. 2, pp. 355-365, 1995.

[4] D. H. Teitelbaum, "Parenteral nutrition-associated cholestasis," Current Opinion in Pediatrics, vol. 9, no. 3, pp. 270-275, 1997.

[5] E. R. Briones and F. L. Iber, "Liver and biliary tract changes and injury associated with total parenteral nutrition: pathogenesis and prevention," Journal of the American College of Nutrition, vol. 14, no. 3, pp. 219-228, 1995.

[6] B. A. Carter and R. J. Shulman, "Mechanisms of disease: update on the molecular etiology and fundamentals of parenteral nutrition associated cholestasis," Nature Clinical Practice Gastroenterology and Hepatology, vol. 4, no. 5, pp. 277-287, 2007.

[7] V. J. Kumpf, "Parenteral nutrition-associated liver disease in adult and pediatric patients," Nutrition in Clinical Practice, vol. 21, no. 3, pp. 279-290, 2006.

[8] A. K. Jain, B. Stoll, D. G. Burrin, J. J. Holst, and D. D. Moore, "Enteral bile acid treatment improves parenteral nutritionrelated liver disease and intestinal mucosal atrophy in neonatal pigs," The American Journal of Physiology: Gastrointestinal and Liver Physiology, vol. 302, no. 2, pp. G218-G224, 2012.

[9] D. A. Kelly, "Intestinal failure-associated liver disease: what do we know today?” Gastroenterology, vol. 130, no. 2, pp. S70-S77, 2006.

[10] D. L. Sigalet, "Short bowel syndrome in infants and children: an overview," Seminars in Pediatric Surgery, vol. 10, no. 2, pp. 49$55,2001$.

[11] P. J. Javid, S. Collier, D. Richardson et al., "The role of enteral nutrition in the reversal of parenteral nutrition-associated liver dysfunction in infants," Journal of Pediatric Surgery, vol. 40, no. 6, pp. 1015-1018, 2005.

[12] R. A. Drongowski and A. G. Coran, "An analysis of factors contributing to the development of total parenteral nutritioninduced cholestasis," Journal of Parenteral and Enteral Nutrition, vol. 13, no. 6, pp. 586-589, 1989.

[13] A. Dilsiz, "Total parenteral nutrition-associated cholestasis in surgical neonates," Turkish Journal of Medical Sciences, vol. 29, pp. 689-692, 1999.

[14] C. S. Erickson, A. J. Barlow, J. F. Pierre et al., "Colonic enteric nervous system analysis during parenteral nutrition," Journal of Surgical Research, vol. 184, no. 1, pp. 132-137, 2013.

[15] A. C. Mosenthal, D. Xu, and E. A. Deitch, "Elemental and intravenous total parenteral nutrition diet-induced gut barrier failure is intestinal site specific and can be prevented by feeding nonfermentable fiber," Critical Care Medicine, vol. 30, no. 2, pp. 396-402, 2002.
[16] M. Ekelund, M. Ekelund, S. S. Qader, M. Hallén, and E. Ekblad, "Effects of total parenteral nutrition on rat enteric nervous system, intestinal morphology, and motility," Journal of Surgical Research, vol. 124, no. 2, pp. 187-193, 2005.

[17] H. Niinikoski, B. Stoll, X. Guan et al., "Onset of small intestinal atrophy is associated with reduced intestinal blood flow in TPNfed neonatal piglets," Journal of Nutrition, vol. 134, no. 6, pp. $1467-1474,2004$

[18] M. Miyata, T. Hata, H. Yamakawa, T. Kagawa, K. Yoshinari, and Y. Yamazoe, "Involvement of multiple elements in FXRmediated transcriptional activation of FGF19," Journal of Steroid Biochemistry and Molecular Biology, vol. 132, no. 1-2, pp. 41-47, 2012.

[19] F. J. Gonzalez, "Nuclear receptor control of enterohepatic circulation," Comprehensive Physiology, vol. 2, no. 4, pp. 28112828, 2012.

[20] M. Makishima, A. Y. Okamoto, J. J. Repa et al., "Identification of a nuclear receptor for bite acids," Science, vol. 284, no. 5418, pp. 1362-1365, 1999.

[21] R. G. J. Visschers, M. D. Luyer, F. G. Schaap, S. W. M. Olde Damink, and P. B. Soeters, "The gut-liver axis," Current Opinion in Clinical Nutrition and Metabolic Care, vol. 16, no. 5, pp. 576581,2013

[22] T. Claudel, B. Staels, and F. Kuipers, "The Farnesoid X receptor: a molecular link between bile acid and lipid and glucose metabolism," Arteriosclerosis, Thrombosis, and Vascular Biology, vol. 25, no. 10, pp. 2020-2031, 2005.

[23] S. Modica, M. Petruzzelli, E. Bellafante et al., "Selective activation of nuclear bile acid receptor FXR in the intestine protects mice against cholestasis," Gastroenterology, vol. 142, no. 2, pp. 355.e4-365.e4, 2012

[24] S. J. L. B. Zweers, K. A. C. Booij, M. Komuta et al., "The human gallbladder secretes fibroblast growth factor 19 into bile: towards defining the role of fibroblast growth factor 19 in the enterobiliary tract," Hepatology, vol. 55, no. 2, pp. 575-583, 2012.

[25] B. Goodwin, S. A. Jones, R. R. Price et al., "A regulatory cascade of the nuclear receptors FXR, SHP-1, and LRH-1 represses bile acid biosynthesis," Molecular Cell, vol. 6, no. 3, pp. 517-526, 2000

[26] T. T. Lu, M. Makishima, J. J. Repa et al., "Molecular basis for feedback regulation of bile acid synthesis by nuclear receptos," Molecular Cell, vol. 6, no. 3, pp. 507-515, 2000.

[27] J. A. Holt, G. Luo, A. N. Billin et al., "Definition of a novel growth factor-dependent signal cascade for the suppression of bile acid biosynthesis," Genes and Development, vol. 17, no. 13, pp. 15811591, 2003.

[28] L. Wang, Y.-K. Lee, D. Bundman et al., "Redundant pathways for negative feedback regulation of bile acid production," Developmental Cell, vol. 2, no. 6, pp. 721-731, 2002.

[29] T. Inagaki, M. Choi, A. Moschetta et al., "Fibroblast growth factor 15 functions as an enterohepatic signal to regulate bile acid homeostasis," Cell Metabolism, vol. 2, no. 4, pp. 217-225, 2005.

[30] T. Lundåsen, C. Gälman, B. Angelin, and M. Rudling, "Circulating intestinal fibroblast growth factor 19 has a pronounced diurnal variation and modulates hepatic bile acid synthesis in man," Journal of Internal Medicine, vol. 260, no. 6, pp. 530-536, 2006.

[31] M.-H. Xie, I. Holcomb, B. Deuel et al., "FGF-19, a novel fibroblast growth factor with unique specificity for FGFR4," Cytokine, vol. 11, no. 10, pp. 729-735, 1999. 
[32] T. Nishimura, Y. Utsunomiya, M. Hoshikawa, H. Ohuchi, and N. Itoh, "Structure and expression of a novel human FGF, FGF19, expressed in the fetal brain," Biochimica et Biophysica Acta, vol. 1444, no. 1, pp. 148-151, 1999.

[33] E. Tomlinson, L. Fu, L. John et al., "Transgenic mice expressing human fibroblast growth factor-19 display increased metabolic rate and decreased adiposity," Endocrinology, vol. 143, no. 5, pp. 1741-1747, 2002.

[34] I. Kim, S.-H. Ahn, T. Inagaki et al., "Differential regulation of bile acid homeostasis by the farnesoid X receptor in liver and intestine," Journal of Lipid Research, vol. 48, no. 12, pp. 26642672, 2007.

[35] L. Fu, L. M. John, S. H. Adams et al., "Fibroblast growth factor 19 increases metabolic rate and reverses dietary and leptindeficient diabetes," Endocrinology, vol. 145, no. 6, pp. 2594-2603, 2004.

[36] S. E. Hughes, "Differential expression of the fibroblast growth factor receptor (FGFR) multigene family in normal human adult tissues," Journal of Histochemistry and Cytochemistry, vol. 45, no. 7, pp. 1005-1019, 1997.

[37] C. Yu, F. Wang, M. Kan et al., "Elevated cholesterol metabolism and bile acid synthesis in mice lacking membrane tyrosine kinase receptor FGFR4," The Journal of Biological Chemistry, vol. 275, no. 20, pp. 15482-15489, 2000.

[38] X. Huang, C. Yang, Y. Luo, C. Jin, F. Wang, and W. L. McKeehan, "FGFR4 prevents hyperlipidemia and insulin resistance but underlies high-fat diet-induced fatty liver," Diabetes, vol. 56, no. 10, pp. 2501-2510, 2007.

[39] J. E. Heubi, D. A. Wiechmann, V. Creutzinger et al., "Tauroursodeoxycholic acid (TUDCA) in the prevention of total parenteral nutrition-associated liver disease," Journal of Pediatrics, vol. 141, no. 2, pp. 237-242, 2002.

[40] E. Reihner, I. Bjorkhem, B. Angelin, S. Ewerth, and K. Einarsson, "Bile acid synthesis in humans: regulation of hepatic microsomal cholesterol $7 \alpha$-hydroxylase activity," Gastroenterology, vol. 97, no. 6, pp. 1498-1505, 1989.

[41] D. J. Parks, S. G. Blanchard, R. K. Bledsoe et al., "Bile acids: natural ligands for an orphan nuclear receptor," Science, vol. 284, no. 5418, pp. 1365-1368, 1999.

[42] A. Lucas, S. R. Bloom, and A. Aynsley Green, "Metabolic and endocrine consequences of depriving preterm infants of enteral nutrition," Acta Paediatrica Scandinavica, vol. 72, no. 2, pp. 245249, 1983.

[43] T. Fedorowski, G. Salen, G. S. Tint, and E. Mosbach, "Transformation of chenodeoxycholic acid and ursodeoxycholic acid by human intestinal bacteria," Gastroenterology, vol. 77, no. 5, pp. 1068-1073, 1979.

[44] C. R. Cole and T. R. Ziegler, "Small bowel bacterial overgrowth: a negative factor in gut adaptation in pediatric SBS," Current Gastroenterology Reports, vol. 9, no. 6, pp. 456-462, 2007.

[45] S. N. Lichtman, R. B. Sartor, J. Keku, and J. H. Schwab, "Hepatic inflammation in rats with experimental small intestinal bacterial overgrowth," Gastroenterology, vol. 98, no. 2, pp. 414-423, 1990.

[46] H. R. Freund, M. Muggia-Sullam, R. LaFrance, E. B. Enrione, M. B. Popp, and H. S. Bjornson, "A possible beneficial effect of metronidazole in reducing TPN-associated liver function derangements," Journal of Surgical Research, vol. 38, no. 4, pp. 356-363, 1985.

[47] J. F. Whiting, R. M. Green, A. B. Rosenbluth, and J. L. Gollan, "Tumor necrosis factor-alpha decreases hepatocyte bile salt uptake and mediates endotoxin-induced cholestasis," Hepatology, vol. 22, no. 4, part 1, pp. 1273-1278, 1995.

[48] A. A. Nanji and F. H. Anderson, "Cholestasis associated with parenteral nutrition develops more commonly with hematologic malignancy than with inflammatory bowel disease," Journal of Parenteral and Enteral Nutrition, vol. 8, no. 3, p. 325, 1984.

[49] H. Fouin-Fortunet, L. Le Quernec, S. Erlinger, E. Lerebours, and R. Colin, "Hepatic alterations during total parenteral nutrition in patients with inflammatory bowel disease: a possible consequence of lithocholate toxicity," Gastroenterology, vol. 82, no. 5, part 1, pp. 932-937, 1982.

[50] Y. J. Zheng, Y. K. Tam, and R. T. Coutts, "Endotoxin and cytokine released during parenteral nutrition," Journal of Parenteral and Enteral Nutrition, vol. 28, no. 3, pp. 163-168, 2004.

[51] E. H. Forrest, K. A. Oien, S. Dickson, D. Galloway, and P. R. Mills, "Improvement in cholestasis associated with total parenteral nutrition after treatment with an antibody against tumour necrosis factor alpha," Liver, vol. 22, no. 4, pp. 317-320, 2002.

[52] M. Trauner, M. Arrese, H. Lee, J. L. Boyer, and S. J. Karpen, "Endotoxin downregulates rat hepatic ntcp gene expression via decreased activity of critical transcription factors," Journal of Clinical Investigation, vol. 101, no. 10, pp. 2092-2100, 1998.

[53] M. Hojo, N. Sano, and H. Takikawa, "Effects of lipopolysaccharide on the biliary excretion of bile acids and organic anions in rats," Journal of Gastroenterology and Hepatology, vol. 18, no. 7, pp. 815-821, 2003.

[54] R. Ghose, T. L. Zimmerman, S. Thevananther, and S. J. Karpen, "Endotoxin leads to rapid subcellular re-localization of hepatic $\mathrm{RXR} \alpha$ : a novel mechanism for reduced hepatic gene expression in inflammation," Nuclear Receptor, vol. 2, article 4, 2004.

[55] T. Inagaki, A. Moschetta, Y.-K. Lee et al., "Regulation of antibacterial defense in the small intestine by the nuclear bile acid receptor," Proceedings of the National Academy of Sciences of the United States of America, vol. 103, no. 10, pp. 3920-3925, 2006.

[56] R. M. Gadaleta, K. J. Van Erpecum, B. Oldenburg et al., "Farnesoid X receptor activation inhibits inflammation and preserves the intestinal barrier in inflammatory bowel disease," Gut, vol. 60, no. 4, pp. 463-472, 2011.

[57] B. A. Carter, O. A. Taylor, D. R. Prendergast et al., "Stigmasterol, a soy lipid-derived phytosterol, is an antagonist of the bile acid nuclear receptor FXR," Pediatric Research, vol. 62, no. 3, pp. 301306, 2007.

[58] J. Fuchs, E. M. Fallon, K. M. Gura, and M. Puder, "Use of an omega-3 fatty acid-based emulsion in the treatment of parenteral nutrition-induced cholestasis in patients with microvillous inclusion disease," Journal of Pediatric Surgery, vol. 46, no. 12, pp. 2376-2382, 2011.

[59] H. E. Lilja, Y. Finkel, M. Paulsson, and S. Lucas, "Prevention and reversal of intestinal failure-associated liver disease in premature infants with short bowel syndrome using intravenous fish oil in combination with omega-6/9 lipid emulsions," Journal of Pediatric Surgery, vol. 46, no. 7, pp. 1361-1367, 2011.

[60] R. S. Venick and K. Calkins, "The impact of intravenous fish oil emulsions on pediatric intestinal failure-associated liver disease," Current Opinion in Organ Transplantation, vol. 16, no. 3, pp. 306-311, 2011.

[61] Y. H. Yu, E. C. Lin, S. C. Wu et al., "Docosahexaenoic acid regulates adipogenic genes in myoblasts via porcine peroxisome 
proliferator-activated receptor gamma," Journal of Animal Science, vol. 86, no. 12, pp. 3385-3392, 2008.

[62] G. L. Størvold, K. G. Fleten, C. G. Olsen, T. Follestad, H. E. Krokan, and S. A. Schønberg, "Docosahexaenoic acid activates some SREBP-2 targets Independent of cholesterol and ER stress in SW620 colon cancer cells," Lipids, vol. 44, no. 8, pp. 673-683, 2009.

[63] G. Howell III, X. Deng, C. Yellaturu et al., "N-3 polyunsaturated fatty acids suppress insulin-induced SREBP-1c transcription via reduced trans-activating capacity of $\mathrm{LXR} \alpha$," Biochimica et Biophysica Acta, vol. 1791, no. 12, pp. 1190-1196, 2009.

[64] N. Tanaka, X. Zhang, E. Sugiyama et al., "Eicosapentaenoic acid improves hepatic steatosis independent of PPAR $\alpha$ activation through inhibition of SREBP-1 maturation in mice," Biochemical Pharmacology, vol. 80, no. 10, pp. 1601-1612, 2010.

[65] A. Zhao, J. Yu, J.-L. Lew, L. Huang, S. D. Wright, and J. Cui, "Polyunsaturated fatty acids are FXR ligands and differentially regulate expression of FXR targets," DNA and Cell Biology, vol. 23, no. 8, pp. 519-526, 2004.

[66] T. Yoshikawa, H. Shimano, N. Yahagi et al., "Polyunsaturated fatty acids suppress sterol regulatory element-binding protein $1 \mathrm{c}$ promoter activity by inhibition of liver X receptor (LXR) binding to LXR response elements," Journal of Biological Chemistry, vol. 277, no. 3, pp. 1705-1711, 2002. 


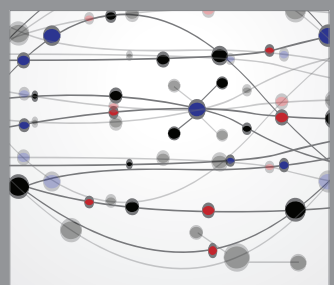

The Scientific World Journal
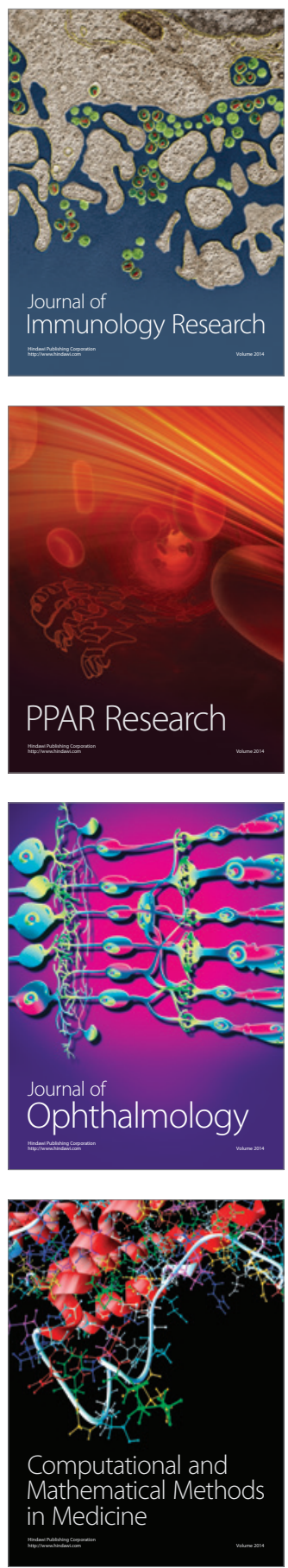

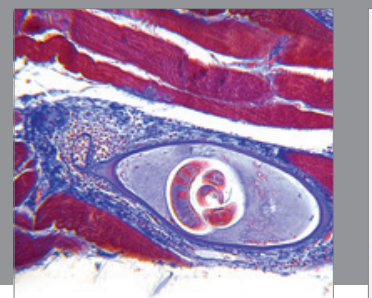

Gastroenterology

Research and Practice
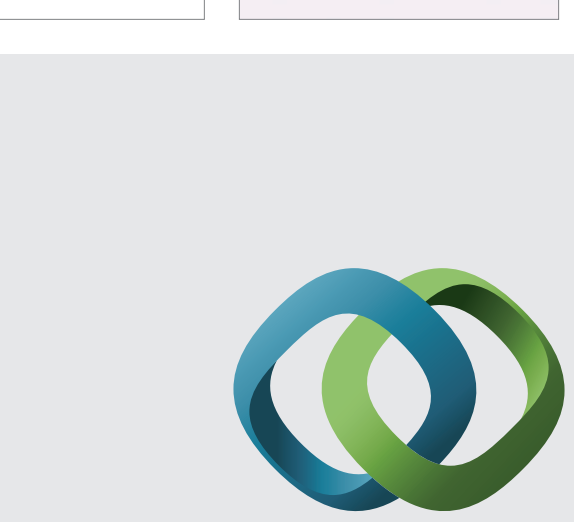

\section{Hindawi}

Submit your manuscripts at

http://www.hindawi.com
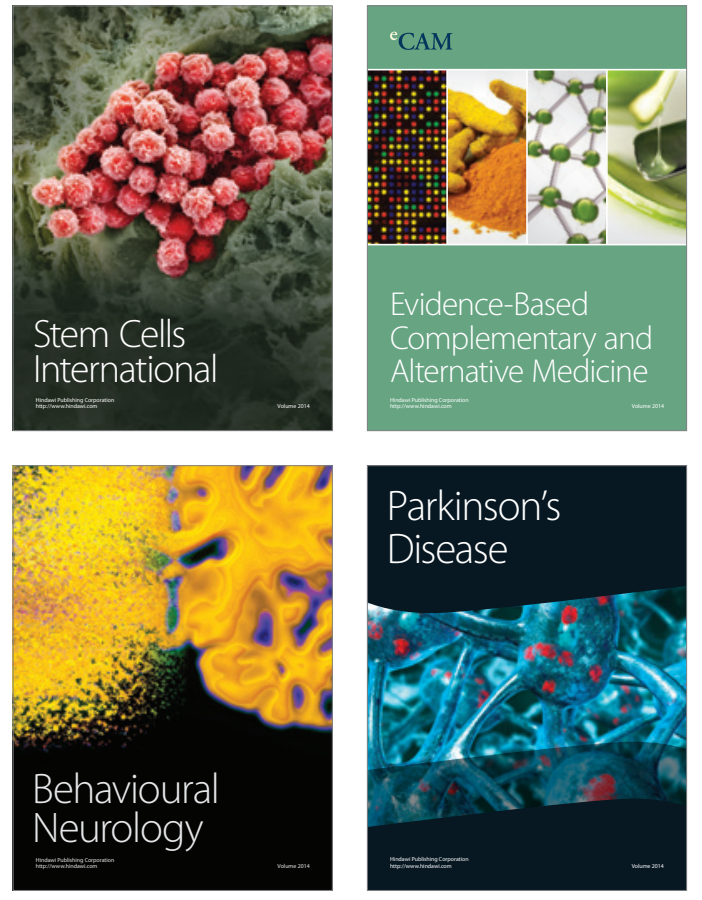
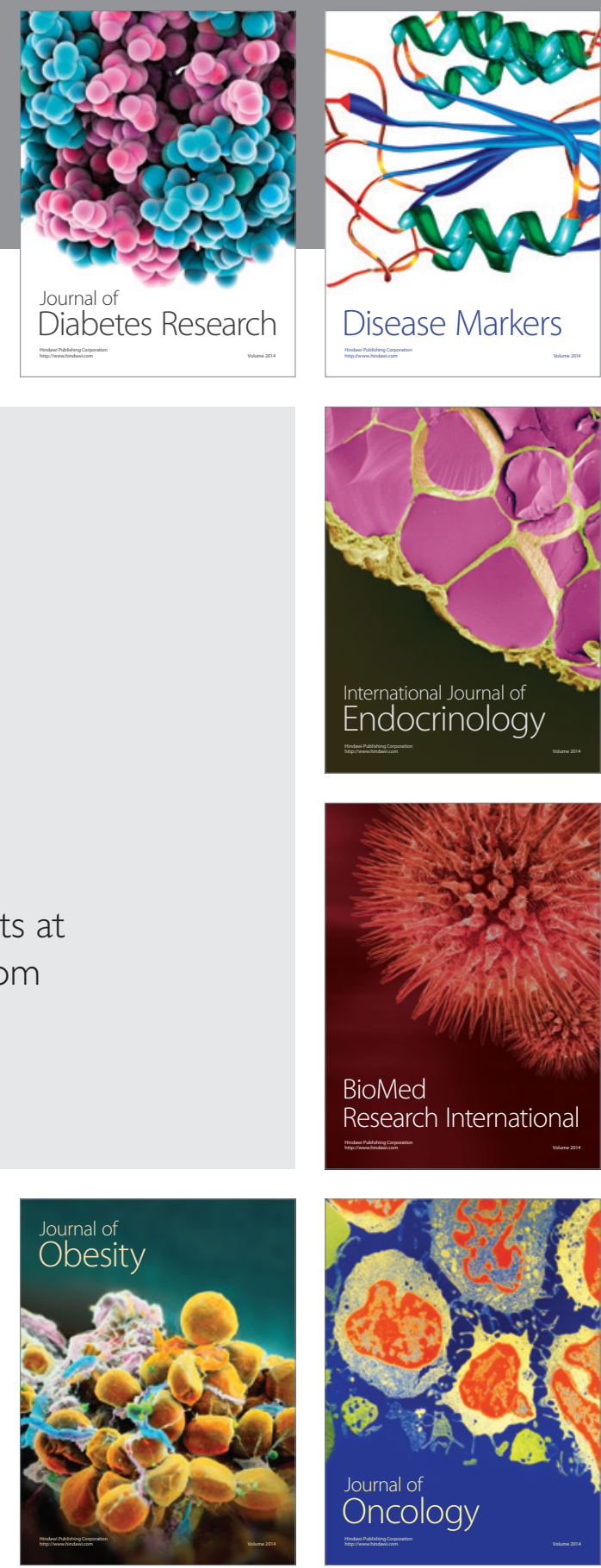

Disease Markers
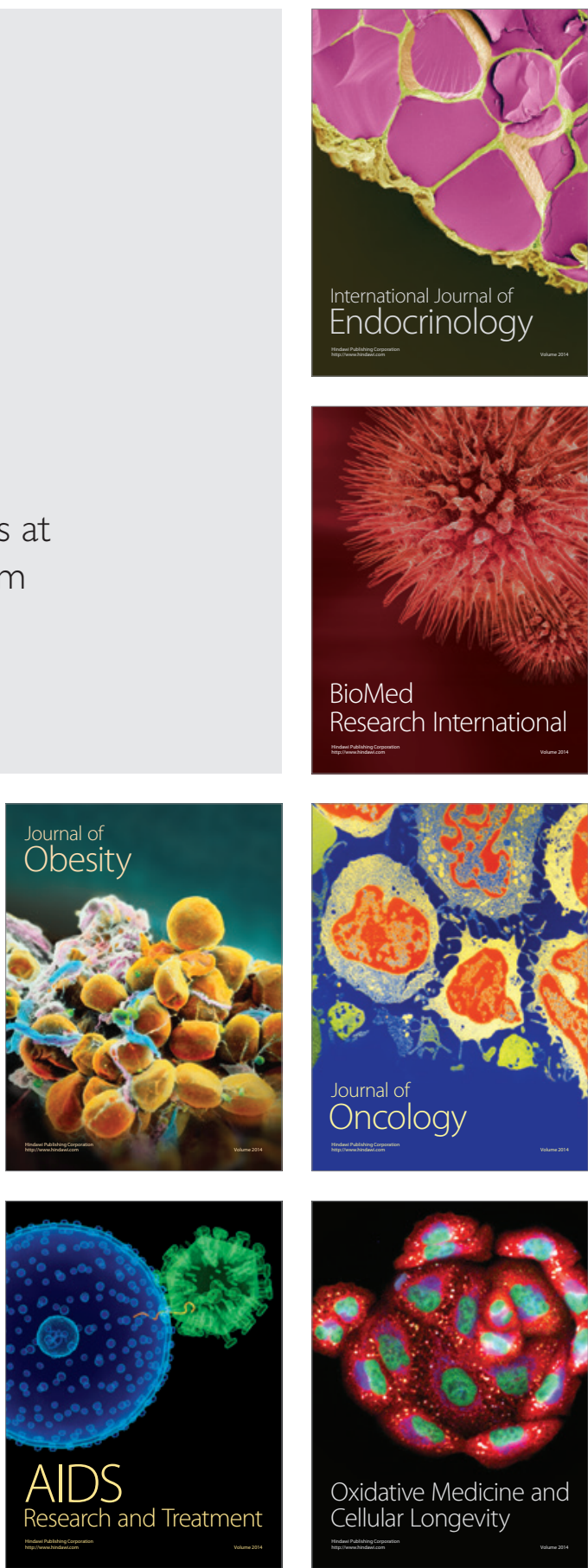